\title{
Aeroelastic instability of cantilevered flexible plates in uniform flow
}

\author{
By CHRISTOPHE ELOY, ROMAIN LAGRANGE, \\ CLAIRE SOUILLIEZ AND LIONEL SCHOUVEILER
}

IRPHE, CNRS \& Aix-Marseille Université, 49 rue Joliot-Curie, 13013 Marseille, France

(Received 2 February 2011)

We address the flutter instability of a flexible plate immersed in an axial flow. This instability is similar to flag flutter and results from the competition between destabilising pressure forces and stabilising bending stiffness. In previous experimental studies, the plates have always appeared much more stable than the predictions of two-dimensional models. This discrepancy is discussed and clarified in this paper by examining experimentally and theoretically the effect of the plate aspect ratio on the instability threshold. We show that the two-dimensional limit cannot be achieved experimentally because hysteretical behaviour and three-dimensional effects appear for plates of large aspect ratio. The nature of the instability bifurcation (sub- or supercritical) is also discussed in the light of recent numerical results.

\section{Introduction}

The flutter of a flexible plate immersed in an axial flow is a canonical example of flowinduced vibrations. This instability can be experienced in everyday life when one observes a flag flapping in the wind. Because this phenomenon appears in many applications (paper industry, airfoil flutter, snoring), it has motivated a large literature which has been recently reviewed by Païdoussis (2004). This instability can be regarded as a competition between fluid forces and elasticity. Indeed, when the plate experiences a small lateral deflection, a destabilising pressure jump can appear across the plate, while the bending stiffness tends to bring the plate back to the stable planar state.

This system can be studied by restricting the analysis to one-dimensional flutter modes as observed in most experiments. In this case, the plate motion obeys the Euler-Bernoulli beam equation with additional pressure forces which are calculated by assuming a potential flow. To simplify the problem further, Shelley et al. (2005) considered a plate infinite in both directions in a similar way to the stability analysis of a jet by Lord Rayleigh (1879) who already noted in his seminal paper the analogy with the problem of flag flutter.

In other theoretical studies, the plate length $L$ (or chord) takes a finite value while two asymptotic limits are considered for its span $H$. If $H \ll L$, the fluid forces can be calculated using the slender body theory of Lighthill (1960) (e.g. Datta \& Gottenberg 1975: Lemaitre et al. 2005) and if $H \gg L$ the problem can be treated as two-dimensional (as done by Kornecki et al. 1976; Huang 1995; Watanabe et al. 2002a: Guo \& Païdoussis 2000). In this latter case, the flow is entirely described by point-vortices which are distributed within the plate and possibly in its wake. It is known from airfoil theory that this problem does not admit a unique solution (intrinsically because the Laplace equation has to be solved on an open domain). Kornecki et al. (1976) used two approaches to 
treat this non-unicity. They first considered that the total circulation around the plate vanishes and thus no vorticity is shed in the wake. Second, they used the Kutta condition applied at the trailing edge to prescribe the circulation around the plate as Theodorsen (1935) did in his study of airfoil flutter (see also Crighton 1985, for an application of the Kutta hypothesis to unsteady flows). The Kutta condition imposes advected vortices in the wake which makes this model more physical for this flag-type instability (see for instance chapter 5-6 of Bisplinghoff et al. 1983). Note that this shed vorticity can equivalently be regarded as the consequence of the Kelvin's circulation theorem. Using these two-dimensional flow models, a stability analysis can be carried out that permits the prediction of the critical velocity for plate flutter.

The first visualisations of the flutter instability have been performed with flags made of various fabrics by Taneda (1968). A few years later, Datta \& Gottenberg (1975) carried out experiments with long ribbons hanging in airflow. This slender body limit $(H \ll L)$ has been reexamined recently by Lemaitre et al. (2005) and results show good agreement with linear stability analysis. For larger aspect ratios, Huang (1995), Yamaguchi et al. (2000) and Watanabe et al. (2002b) have considered both the effects of the plate length and of the material properties on the flutter instability. However, to the our knowledge, the effect of aspect ratio has not been investigated up to now.

Cantilevered plates in axial flow have also been modelled numerically. Watanabe et al. (2002a) and Balint \& Lucey (2005) used a two-dimensional Navier-Stokes solver combined to a linear beam model for the plate. The critical velocities found in these studies agree with the results of two-dimensional stability analysis. The nature of the bifurcation in this flutter instability has also been studied using a nonlinear beam equation for the plate and two-dimensional (Tang \& Païdoussis 2007) or three-dimensional (Tang et al. 2003) vortex methods to model the potential flow. Similarly to the slender body limit (Yadykin et al. 2001), the bifurcation is shown to be supercritical in these models. However, Alben \& Shelley (2008) recently found hysteresis and bistability using a two-dimensional model taking into account the nonlinearities originating both from the flow and from the elastic plate. Hysteresis has also been reported in previous experiments with an hysteresis loop at least an order of magnitude wider than in the simulations of Alben \& Shelley (2008). This indicates a subcritical bifurcation. To date, the real nature of the bifurcation and the apparent discrepancy between most numerical models and the experiments remains unexplained.

The results of existing two-dimensional models have been compiled by Watanabe $\mathrm{et}$ al. (2002a) and compared with existing experimental data (see also the recent comparison made by Tang \& Païdoussis 2007). Remarkably all these theories predict approximately the same critical velocities but strongly underestimate the measured thresholds. In other words, the plate appears systematically more stable than predicted by a two-dimensional approximation. This discrepancy has motivated the recent study of Eloy et al. (2007) in which the finite plate span is explicitly taken into account in the analysis. Shayo (1980) already addressed the effect of aspect ratio on the flutter instability. However, he made several mathematical assumptions to simplify the stability analysis which led him to falsely conclude that the larger the aspect ratio is the more stable is the system. The present paper aims at comparing the predictions of Eloy et al. (2007) with experimental measurements in which the effect of the plate aspect ratio is extensively investigated.

This paper is divided as follows: in $\S 2$ the physical model is presented and the dimensionless parameters are introduced; in $\S 3$ the experimental setup is briefly described and the main results are given in $\S 4$ finally these results are discussed in the light of previous theoretical and numerical models in $\S 5$. 

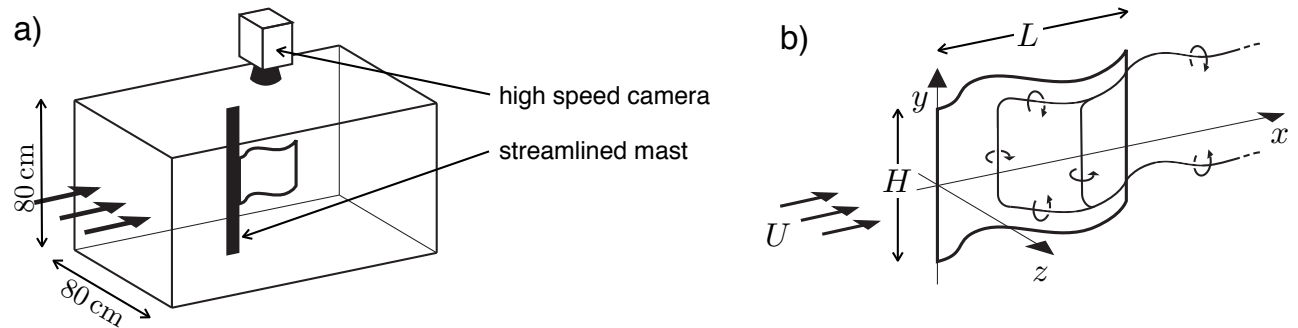

FigurE 1. Sketches of the experimental setup (a) and of the flexible plate subject to the one-dimensional deflection $z(x, t)(\mathrm{b})$.

\section{Physical model}

As shown in figure 1, we consider a flexible plate of span $H$ and length $L$, lying in the vertical plane $(O x y)$ and immersed in an axial flow of velocity $U$. Its flexural rigidity is given by $D=E h^{3} / 12\left(1-\nu^{2}\right)$, where $E$ is the Young's modulus, $h$ the plate thickness and $\nu$ its Poisson's ratio. For small lateral deflections $z(x, t)$, the plate motion is driven by the linearised Euler-Bernoulli equation

$$
m \partial_{t}^{2} z+D \partial_{x}^{4} z+\langle p\rangle=0
$$

where $m$ is the mass per unit surface of the plate, $p(x, y, t)$ is the pressure jump across the plate and the notation $\langle$.$\rangle stands for the average along the span H$. Equation (2.1) is valid in the limit of an inviscid fluid and for a material of negligible visco-elastic damping. In addition the deflection $z$ satisfies clamped boundary conditions at the leading edge: $z(0)=\partial_{x} z(0)=0$ and free boundary conditions at the trailing edge: $\partial_{x}^{2} z(L)=\partial_{x}^{3} z(L)=$ 0 .

Assuming an inviscid flow, the perturbation velocity can be fully described by the vorticity distribution in the flow. Vortex-lines are located in the plate and in its wake as sketched in figure 1(b). The pressure jump across the plate only depends on the $y$ component of the vorticity distribution $\gamma(x, y, t)$ through the unsteady Bernoulli equation

$$
\partial_{x} p=\rho\left(\partial_{t}+U \partial_{x}\right) \gamma
$$

where $\rho$ is the fluid density and $\gamma$ has the dimension of a velocity (i.e. a circulation per unit length). As seen from this equation a vorticity distribution of the form $\gamma_{0}(y) \exp [\mathrm{i}(k x-$ $\omega t)$ ] with wave velocity $\omega / k=U$ is compatible with a zero pressure jump and is indeed the distribution of vorticity in the wake for small plate deflections.

In order to couple the vorticity distribution $\gamma$ to the plate deflection $z$, a kinematic condition is enforced on the plate surface which can be written as

$$
\oint\langle\gamma(\xi)\rangle K(x-\xi) \mathrm{d} \xi=\left(\partial_{t}+U \partial_{x}\right) z=w(x, t)
$$

where the function $K$ is a kernel defined below, $\xi$ is a dummy variable in place of $x$, $w$ is the normal plate velocity in the fluid reference frame, the brackets $\langle$.$\rangle still denote$ averaging along $H$ and the letter $C$ across the integral sign indicates that the Cauchy principal value should be taken. Since this inverse problem can have non-unique solutions, the Kutta condition is also used at the trailing edge i.e. $p\left(L^{-}\right)=0$. The kernel $K$ in $(2.3)$ expresses the influence of a vertical vorticity line located in $\xi$ on the $z$-component of the fluid velocity in $x$. For an infinite span, the azimuthal velocity of a point-vortex in two dimensions yields the kernel $K(X)=1 / 2 \pi X$ used in all the two-dimensional models. For an asymptotically small $H$ the Lighthill's slender body theory is equivalent to taking $K(X)=\operatorname{sgn}(X) / \pi H$. In Eloy et al. (2007), we have shown that an approximate kernel 
can be used for any $H$

$$
K(X)=\frac{1}{2 \pi X}+\left(\frac{1}{\pi H}-\frac{1 / \pi-1 / 8}{H+|X|}\right) \operatorname{sgn}(X) .
$$

This kernel is constructed as a composite of the correct asymptotic expansions for small and large $H$. Morevover, for all $X$, it has been shown to be within $2 \%$ of the exact kernel which cannot be expressed analytically.

Applying the operator $\rho\left(\partial_{t}+U \partial_{x}\right)$ on 2.3 and integrating by parts yields the following inverse problem for the pressure jump

$$
\oint\left\langle\partial_{\xi} p(\xi)\right\rangle K(x-\xi) \mathrm{d} \xi=\rho\left(\partial_{t}+U \partial_{x}\right)^{2} z=\rho\left(\partial_{t}+U \partial_{x}\right) w .
$$

This Fredholm equation of the first kind has been solved in Eloy et al. (2007) to address the flutter instability of the plate. But, since an integration by parts was performed to obtain (2.5), the pressure jump is implicitly assumed to be non-singular at leading and trailing edges. At the trailing-edge, this assumption is correct because the singularity vanishes thanks to the Kutta condition. However, one expects an inverse square-root pressure singularity at the leading-edge. As we shall see in $\S 4$, the instability characteristics are not greatly modified by the treatment of this leading edge singularity. It is probably due to the fact that it corresponds to the clamped end of the plate where pressure forces do no work. The model derived from Eloy et al. (2007) will be called model without singularity in the rest of the paper.

To assess the validity of this model and to allow comparisons with the two-dimensional model of Kornecki et al. (1976), another approach has been used in the present paper. It is valid in the limit of large aspect ratio and it will be referred to as the asymptotic model. In this model, the two leading order terms have been retained in 2.4 leading to the following kernel

$$
K(X)=\frac{1}{2 \pi X}+\frac{1}{8 H} \operatorname{sgn}(X)+O\left(H^{-2}\right) .
$$

Note that we have shown in Eloy et al. (2007) that this is the mathematically correct expansion for $H \gg|X|$. The problem being linear by construction, the stability of this fluid-structure coupling can be now addressed assuming a Galerkin decomposition for the deflection

$$
z(x, t)=\sum_{n=1}^{\infty} a_{n} z_{n}(x) \mathrm{e}^{\mathrm{i} \omega t}
$$

where the $z_{n}$ are the orthogonal eigenmodes of a beam in vacuo thus satisfying the proper boundary conditions. For a given Galerkin mode, one has then to invert (2.3) with $K$ given by (2.6) and insert the solution in (2.2) to find the corresponding pressure jump $\left\langle p_{n}\right\rangle \exp (\mathrm{i} \omega t)$. This procedure allows the leading edge singularity to be taken into account properly. The partial differential equation (2.1) is then reduced to an eigenvalue problem for the complex frequency $\omega$ whose eigenmodes correspond to the instability modes. For small flow velocities, all modes are stable (i.e. their complex frequencies $\omega$ have a positive imaginary part). When $U$ is larger than a critical flow velocity $U_{c}$, one of the instability modes eventually becomes unstable.

Using $L$ and $L / U$ as characteristic length and time, the system parameters are reduced to three dimensionless numbers: the reduced velocity $U^{*}$, the mass ratio $M^{*}$ and the aspect ratio $H^{*}$ given by

$$
U^{*}=L U \sqrt{m / D}, \quad M^{*}=\rho L / m, \quad H^{*}=H / L .
$$


With these dimensionless parameters, the present 'asymptotic model' and the 'model without singularity' detailed in Eloy et al. (2007) permit the prediction of the critical velocity $U_{c}^{*}\left(M^{*}, H^{*}\right)$ and the dimensionless mode frequencies $\omega^{*}=\omega L / U$. The results of the two-dimensional model of Kornecki et al. (1976) have also been recalculated in the present paper with better computer accuracy to allow comparisons. It is strictly equivalent to the 'asymptotic model' for infinite span $H$.

\section{Experimental setup}

As sketched in figure 1(a) experiments were performed in a low-turbulence closed wind tunnel of $80 \times 80 \mathrm{~cm}^{2}$ cross section. The wind velocity $U$ could be varied continuously up to $65 \mathrm{~m} \mathrm{~s}^{-1}$ and was measured with a Pitot tube. The plates were clamped in a streamlined mast crossing the wind tunnel vertically. To ensure that the mast had negligible effects on the instability, two masts of different cross section were used (mast 1: thickness $\times$ chord $=$ $4 \mathrm{~mm} \times 20 \mathrm{~mm}$; mast 2: $9 \mathrm{~mm} \times 43 \mathrm{~mm}$ ). Plates were cut in Mylar sheets of mass per unit area $m=0.14 \mathrm{~kg} \mathrm{~m}^{-2}$ and flexural rigidity $D=0.48 \times 10^{-3} \mathrm{Nm}$. To estimate $D$, the deflection due to gravity was measured for horizontally clamped Mylar strips of various lengths. The plate length $L$ was varied between 2 and $30 \mathrm{~cm}$ for a thickness $h=0.16 \mathrm{~mm}$.

In all experiments presented in this paper, the same protocol has been followed. The plate is clamped in the mast and the flow velocity is slowly increased starting from zero. At small velocities the plate appears stable, i.e. steady and aligned with the flow. Eventually, for a critical flow velocity $U_{c}^{*}$, the plate flutters spontaneously with a large amplitude and a well-defined frequency. Then the flow velocity is slowly decreased in small decrements until the plate returns to its stable state again at another critical velocity $U_{d}^{*}$ as illustrated in figure 2 .

Visualisations were carried out through the top wall of the wind tunnel with a high speed video camera aligned with the $y$-axis (see figure 1 a). The top edge of the plate was painted white and illuminated to record its motion and aid measuring the flutter frequency. In the present experiments, the camera was operating at $300 \mathrm{~Hz}$ with a $512 \times 512$ pixel resolution and the exposure time was varied between 300 and $1500 \mu \mathrm{s}$. The camera visualisations are used to extract the flutter amplitude $A$ and its angular frequency $\omega^{*}$. This is done by detecting in each snapshot of the plate deflection at $3 / 4$ of the total plate length.

\section{Results}

The flutter amplitude $A$ is plotted in figure 2 as a function of the reduced velocity; this plot exhibits a hysteretic behaviour. This has been observed in all experiments with sufficiently large aspect ratio $\left(H^{*} \gtrsim 1\right)$. This hysteresis together with the large mode amplitude observed at threshold evokes a subcritical bifurcation as recently suggested by the numerical results of Alben \& Shelley (2008). Note however that the wideness of the hysteresis loop observed in the present experiments is of the order of $20 \%$ (it is defined as $\left.\left(U_{c}^{*}-U_{d}^{*}\right) / U_{c}^{*}\right)$ whereas it is only of the order of $1 \%$ in the simulations of Alben \& Shelley (2008). In other numerical models (Yadykin et al. 2001: Tang et al. |2003, Tang \& Païdoussis 2007), the bifurcation is found to be supercritical. Figure 2 shows that the amplitudes near threshold can be reasonably well fitted with a square-root law giving evidence that the bifurcation could indeed be supercritical and that the observed hysteresis could just be an artifact as discussed below.

The critical velocities $U_{c}^{*}$ and $U_{d}^{*}$ are plotted in figure 3 as a function of the aspect ratio $H^{*}$ and the mass ratio $M^{*}$. First the plate span is varied with the plate length remaining 


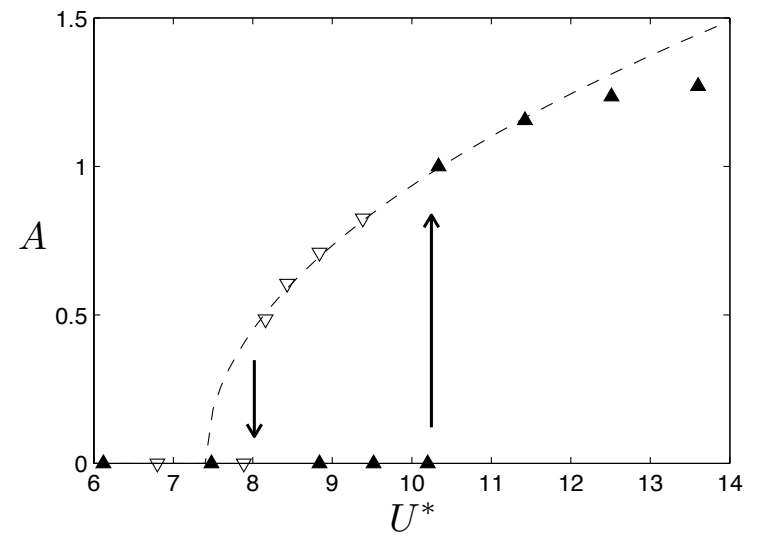

Figure 2. Mode amplitude $A$ (in arbitrary units) as a function of the reduced velocity $U^{*}$. Amplitude is plotted as filled triangles when velocity is increased and open triangles when it is decreased. The experimental parameters are $H^{*}=1$ and $M^{*}=0.6$. The instability exhibits a strong hysteresis with $U_{c}^{*}=10.3$ and $U_{d}^{*}=8$. However, the amplitudes are well fitted by the square-root law $A=58\left(U^{*}-7.4\right)^{1 / 2}$ (dashed line) suggesting a supercritical bifurcation.

constant. This allows the aspect ratio to be varied without changing the other parameters. As expected from slender body theory, the critical velocity tends to decrease as aspect ratio is increased. From figure 3(a) two regimes can be distinguished. For small aspect ratio $\left(H^{*} \lesssim 1\right)$, the measured critical velocities are well predicted by the linear stability analysis and the hysteresis is very small if not absent. For larger aspect ratio $\left(H^{*} \gtrsim 1\right)$ the hysteresis greatly increases. The experimental data points are also more scattered. For these large aspect ratios, the linear stability analysis tends to underestimate the critical velocity $U_{c}^{*}$. Moreover, this threshold seems to slightly increase for $1<H^{*}<3$ contrarily to the decreasing threshold $U_{d}^{*}$ which remains close to the predictions.

The figure 3(a) also permits to quantify the error made by the 'model without singularity'. Indeed it can be seen that it does not exactly converge to the two-dimensional limit when aspect ratio tends to infinity but slightly below. As explained above, this is because in this model the pressure jump is assumed to be regular at the leading edge whereas, in the 'asymptotic model' and in the two-dimensional model of Kornecki et al. (1976), the pressure jump has the physically correct inverse square root singularity.

In figure 3(b), the aspect ratio has been kept constant $\left(H^{*}=1\right)$ by varying by the same factor the plate length and span. The measured critical velocity $U_{c}^{*}$ is compared to the present theoretical predictions and to the two-dimensional model of Kornecki et al. (1976). Experimental data from Huang (1995) are also reported even if these experiments have been carried out with plates spanning on the entire tunnel width with a $2 \mathrm{~mm}$ clearance. This experimental setup was expected to model a two-dimensional flow. In the present analysis, the finiteness of the span is taken into account leading to a smaller pressure jump $\langle p\rangle$ in 2.1 compared to the two-dimensional model and thus a better prediction of the instability threshold. The three models permit to predict different instability modes as $M^{*}$ is increased as illustrated by the different lobes in figure 3(b). If modes are numbered by order of ascending frequencies, the mode two is observed for the smallest mass ratios $\left(M^{*} \lesssim 1.5\right)$. This single-neck mode is pictured in figure $4(\mathrm{a}, \mathrm{b})$. For larger mass ratios $\left(1.5 \lesssim M^{*} \lesssim 5\right)$, the mode three, a double-neck mode, is the first unstable one as illustrated in figure $4(\mathrm{c}, \mathrm{d})$, and for even larger mass ratios $\left(M^{*} \gtrsim 5\right)$, one expect to observe higher order modes. Note that the mode one (the mode with the smallest frequency) is never unstable for a clamped-free plate and accordingly never ob- 

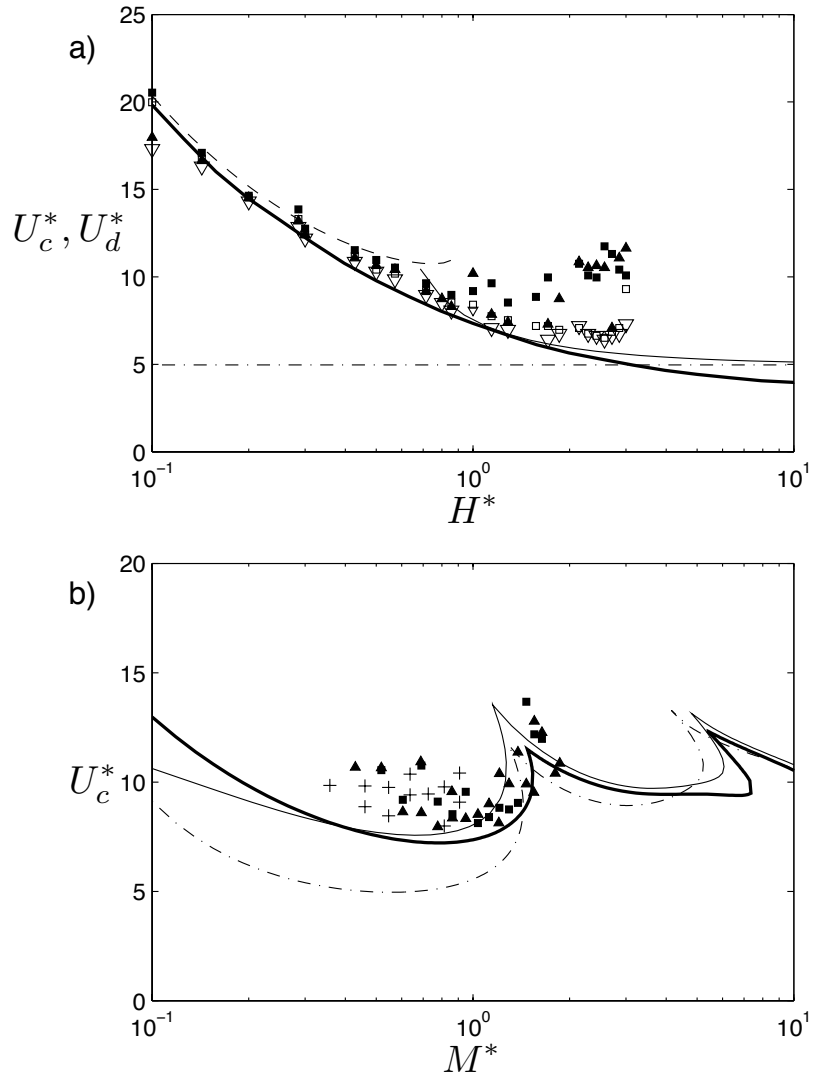

Figure 3. Critical velocities $U_{c}^{*}$ and $U_{d}^{*}$ as a function of the aspect ratio $H^{*}$ for $M^{*}=0.6$ (a) and as a function of the mass ratio $M^{*}$ for $H^{*}=1(\mathrm{~b})$. Filled squares and triangles correspond to measured $U_{c}^{*}$ for the masts 1 and 2 respectively and the same open symbols refer to $U_{d}^{*}$. Crosses correspond to the experiments of Huang (1995) for $0.6<H^{*}<1.5$. The thick solid line is the three-dimensional 'model without singularity' (Eloy et al. 2007) and the thin solid line is the 'asymptotic model' of the present paper for the same parameters. The dashed line is the slender body theory (similar to Lemaitre et al. 2005) and the dash-dotted line corresponds to the two-dimensional theory with circulation (same as Kornecki et al. 1976, with better computer accuracy).

served in the experiments (as discussed in Guo \& Païdoussis 2000). In other words, as $M^{*}$ increases, the typical mode wavelength $\lambda$ is a smaller fraction of the plate length $L$ and therefore if the aspect ratio is kept constant, $H / \lambda$ increases. This span to wavelength ratio has to be large for a two-dimensional theory to be valid and this explain why the difference between the results of the two- and three-dimensional theories reduces as $M^{*}$ increases in figure $3(b)$.

In figure $4(\mathrm{a}, \mathrm{c})$, superimposed views of the plate recorded with the camera during one flutter period are shown. These visualisations were carried out at the instability threshold $U_{c}^{*}$ for plates of same aspect ratio $H^{*}=1$ but of two different mass ratios. As predicted by the analysis, two different modes are observed as $M^{*}$ varies. From these data, the mode shapes and their frequencies can be compared to the theoretical predictions for the same parameters. However nonlinear effects are probably important given the large amplitude observed at threshold and the agreement with the results of the linear stability analysis are only qualitative. 


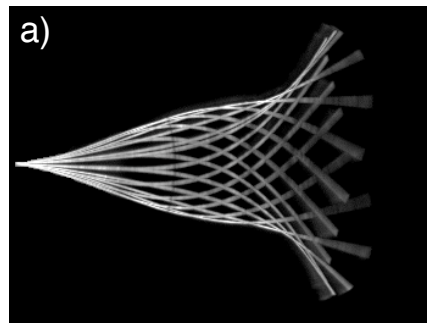

b)
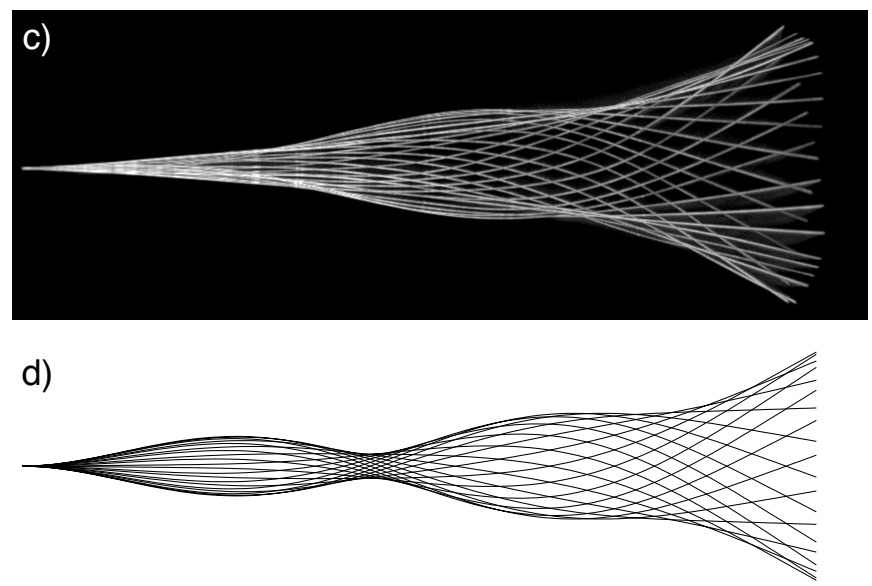

FiguRE 4. Superimposed views of the flutter modes for two plates of same aspect ratio $H^{*}=1$ but with different sizes $\left[M^{*}=0.74\right.$ for (a) and (b), $M^{*}=1.94$ for (c) and (d)]. These amplitudes are obtained at threshold and the measured frequencies are $\omega^{*}=1.82$ for $U_{c}^{*}=8.1$ (a) and $\omega^{*}=2.5$ for $U_{c}^{*}=10.9$ (c). The experimental snapshots are compared with the modes predicted by the present linear stability analysis (with arbitrary amplitude): $\omega^{*}=2.4$ for $U_{c}^{*}=7.2$ (b) and $\omega^{*}=3.7$ for $U_{c}^{*}=10.4(\mathrm{~d})$.

\section{Discussion}

In this paper, we have studied experimentally and theoretically the flutter instability of cantilevered flexible plates in uniform flow. We have shown in particular that the three-dimensionality of the flow has to be taken into account to predict accurately the instability threshold as the plate aspect ratio is varied. We have also shown that hysteresis is present for plates of large aspect ratio and that the instability threshold measured when increasing the velocity does not converge towards the two-dimensional limit as aspect ratio tends to infinity. These experimental results are now discussed with regard to the assumptions made in the stability analysis.

In the experiments with large aspect ratio $\left(H^{*} \gtrsim 2\right)$, we have observed that the flutter mode is no longer purely one-dimensional as the top corner flutters with a larger amplitude than the rest of the plate. Moreover, in some experiments with a very large aspect ratio $\left(H^{*}=4\right)$ and for flow velocity just below threshold, the same top corner flutters while the rest of the plate is still motionless. These two-dimensional motions indicate that the Euler-Bernoulli beam equation is no longer valid for the plate and one should use the nonlinear Föppl-von Kármán equations as soon as the deflection along the span is of the order of the plate thickness (see for instance Landau \& Lifschitz 1986). Several reasons may account for the two-dimensional deflections of the plate. First, the pressure field over the plate is non uniform along the span and exhibit a maximum at mid-span and zeros at the plate edges. Second, the gravity field induces a non trivial stress tensor in the plate that results in compression at the top corner and tension at the bottom 
corner. Since tension has a stabilising effect, this may explain why the plate deflection exhibits a larger amplitude at the top corner for large aspect ratios.

The two-dimensional plate deflections can also originate from small imperfections in the flow caused by the wind tunnel or the mast wake. These imperfections would create flow unsteadiness and two-dimensional plate vibrations could be forced. A small deflection along the span could also be caused by imperfections of the plate clamping or planarity. But whatever their origin, as soon as these two-dimensional deflections or vibrations along the span are of the order of the plate thickness, they act as a stiffening effect. Gauss curvature being energetically costly, once plate is bent along its span, it will bend more difficultly along its length and the instability will be delayed. However, once the plate is unstable, its flutter mode can be purely one-dimensional, flattening any bending along the span, thus generating a hysteresis loop. This could be an elegant explanation for the appearance of the hysteresis loop only for aspect ratios larger than one. Indeed, in this case, the elastic energy required to bend the plate along its span becomes smaller than the energy to bend it along its length. It is then reasonable to think that any defect may trigger deflections along the span of the order of the plate thickness and thus hysteresis.

If one assumes that hysteresis is due to two-dimensional plate deflections and that the bifurcation is supercritical, the decreasing threshold $U_{d}^{*}$ should converge to the present theoretical predictions (based on one-dimensional flutter modes). This is indeed what is observed in figure 3(a) giving another argument for a supercritical bifurcation.

Undesired two-dimensional plate deflections can also be driven by gravity for very long or very light plates. This is what happens when the mass ratio is too large causing the plate to sag, twist or bend under its own weight. In the present study, these undesired effects occur for $M^{*} \gtrsim 5$ and this is why the analysis has been restricted to $M^{*}<2$. When these effects are present however, the instability threshold cannot be predicted by simple means and this presumably explains why the critical velocity measured in the experiments of Watanabe et al. (2002b) and Yamaguchi et al. (2000) for very large $M^{*}$ is about an order of magnitude larger than the predictions of a two-dimensional model.

In this paper, we have argued that the hysteresis loop could be caused by (undesired) two-dimensional plate deflections. We have also suggested that the bifurcation could be supercritical contrary to most experimental works found in the literature. Our arguments are not solid proof however, and the nature of the bifurcation remains an open question that still need further studies.

The authors acknowledge support from the French ANR (No. ANR-06-JCJC-0087).

\section{REFERENCES}

Alben, S. \& Shelley, M. J. 2008 Flapping states of a flag in an inviscid fluid: Bistability and the transition to chaos. Phys. Rev. Lett. 100, 074301.

Balint, T. S. \& Lucey, A. D. 2005 Instability of a cantilevered flexible plate in viscous channel flow. J. Fluids Struct. 20, 893-912.

Bisplinghoff, R. L., Ashley, H. \& Halfman, R. L. 1983 Aeroelasticity. Dover, New York.

Crighton, D. G. 1985 The Kutta condition in unsteady flow. Ann. Rev. Fluid Mech. 17, 411-445.

Datta, S. K. \& Gottenberg, W. G. 1975 Instability of an elastic strip hanging in an airstream. J. Appl. Mech. pp. 195-198.

Eloy, C., Soullliez, C. \& Schouveiler, L. 2007 Flutter of a rectangular plate. J. Fluids Struct. 23, 904-919.

Guo, C. Q. \& PAÏDoussis, M. P. 2000 Stability of rectangular plates with free side-edges in two-dimensional inviscid channel flow. J. Appl. Mech. 67, 171-176. 
HuAng, L. 1995 Flutter of cantilevered plates in axial flow. J. Fluids Struct. 9, 127-147.

Kornecki, A., Dowell, E. H. \& O'Brien, J. 1976 On the aeroelastic instability of twodimensional panels in uniform incompressible flow. J. Sound Vib. 47 (2), 163-178.

Landau, L. D. \& Lifschitz, E. M. 1986 Theory of elasticity. Pergamon Press, New York.

Lemaitre, C., Hémon, P. \& DE Langre, E. 2005 Instability of a long ribbon hanging in axial air flow. J. Fluids Struct. 20 (7), 913-925.

Lighthill, M. J. 1960 Note on the swimming of slender fish. J. Fluid Mech. 9, 305-317.

PAÏDoussis, M. P. 2004 Fluid-Structure Interactions: Slender Structures And Axial Flow, Volume 2. Elsevier Academic Press.

Rayleigh, (Lord) 1879 On the instability of jets. Proc. London Math. Soc. X, 4-13.

Shayo, L. W. 1980 The stability of cantilever panels in uniform incompressible flow. Journal of Sound and Vibration 68 (3), 341-350.

Shelley, M., Vandenberghe, N. \& Zhang, J. 2005 Heavy flags undergo spontaneous oscillations in flowing water. Phys. Rev. Lett. 94, 094302.

TAnedA, S 1968 Waving motions of flags. J. Phys. Soc. Jpn. 24 (2), 392-401.

Tang, D. M., Yamamoto, H. \& Dowell, E. H. 2003 Flutter and limit cycle oscillations of two-dimensional panels in three-dimensional axial flow. J. Fluids Struct. 17, 225-242.

TANG, L. \& PAÏDOussis, M. P. 2007 On the instability and the post-critical behaviour of two-dimensional cantilevered flexible plates in axial flow. J. Sound Vib. 305, 97-115.

Theodorsen, T. 1935 General theory of aerodynamic instability and the mechanism of flutter. Tech. Rep. TR-496. NACA.

Watanabe, Y., Isogai, K., Suzuki, S. \& Sugihara $2002 a$ A theoretical study of paper flutter. J. Fluids Struct. 16 (4), 543-560.

Watanabe, Y., Suzuki, S., Sugihara, M. \& Sueoka, Y. $2002 b$ An experimental study of paper flutter. J. Fluids Struct. 16 (4), 529-542.

Yadykin, Y., Tenetov, V. \& Levin, D. 2001 The flow-induced vibration of a flexible strip hanging vertically in a parallel flow, part 1: temporal aeroelastic instability. J. Fluids Struct. 15, 1167-1185.

Yamaguchi, N., Sekiguchi, T., Yokota, K. \& Tsujimoto, Y. 2000 Flutter limits and behavior of a flexible thin sheet in high-speed flow - II: Experimental results and predicted behaviors for low mass ratios. J. Fluids Eng. 122, 74-83. 